\title{
Leitlinien für Forschungsberichte: Eine Anwendung des CONSORT 2010 Statements
}

\section{A. Ziegler \\ I. R. König}

Medizinisches Publizieren

Schlüsselwörter

CONSORT 2010

- Fachartikel

Kritisches Lesen

Leitlinien für Forschungsberichte

Keywords

- CONSORT 2010

critical appraisal

research publications

reporting guidelines
Institut

Institut für Medizinische

Biometrie und Statistik,

Universität zu Lübeck

\section{Bibliografie}

DoI $10.1055 / \mathrm{s}-0031-1272977$

Dtsch Med Wochenschr 2011;

136: e2-e8 - (c) Georg Thieme

Verlag KG Stuttgart · New York

ISSN 0012-0472

\section{Korrespondenz}

Prof. Dr. rer. nat. Andreas Ziegler

Institut für Medizinische

Biometrie und Statistik,

Universität zu Lübeck,

Universitätsklinikum Schleswig-

Holstein, Campus Lübeck

Maria-Goeppert-Str. 1

23538 Lübeck

Tel. 0451-500/2780

Fax 0451-500/2999

eMail

ziegler@imbs.uni-luebeck.de

\section{Einleitung}

$\checkmark$

Vollständig, klar und transparent muss der Bericht über die Ergebnisse einer Studie sein, damit diese Studie vom Leser genau beurteilt werden kann [5]. Die Elemente, die für die Beurteilung der Qualität und der Resultate zentral sind, werden in Leitlinien für Forschungsberichte zusammengefasst. Das CONSORT Statement (Consolidated Statement of Reporting Trials; konsolidierte Leitlinie zum Berichten randomisierter Studien im Parallelgruppendesign) kann als „Ur-Leitlinie“ für Forschungsberichte angesehen werden [8]. Die Aktualisierung CONSORT 2010 besteht aus einer 25-PunkteCheckliste sowie einem Flussdiagramm und ist eine Leitlinie für Berichte randomisierter Studien jeglicher Art. Allerdings wird darin auf das häufigste Studiendesign, individuell randomisierte Parallelgruppenvergleiche mit zwei Parallelgruppen fokussiert. Andere Studientypen wie Cluster-randomisierte Studien erfordern ein unterschiedliches Maß zusätzlicher Informationen. Diese sind in entsprechenden Erweiterungen verfügbar, welche von www.consort-statement.org heruntergeladen werden können. Diese Erweiterungen stehen allerdings nicht in deutscher Sprache zur Verfügung.

Die Leitlinien erfüllen zwei Aufgaben: Zum einen helfen sie Autoren von Fachartikeln, vollständige und transparente Berichte zu erstellen. Zum anderen können Leser Leitlinien-Elemente zur Einschätzung der Qualität einer publizierten Studie verwenden. Allerdings muss bei der kritischen Beurteilung eines Artikels stets der Unterschied zwischen der Qualität eines Artikels und der eigentliche Studie beachtet werden [5].

Hier wird zur Illustration der zweiten Aufgabe die neue 25-Punkte-Checkliste des CONSORT 2010 Statements auf die Publikation von Richter et al. angewendet [7]. Zusätzlich wird untersucht, inwieweit die Zusammenfassung dieser Veröffentlichung der Erweiterung des CONSORT Statements auf Zusammenfassungen in Zeitschriftenartikeln und Tagungsbänden entspricht $[2,3]$.

\section{Material und Methoden $\nabla$}

Ausgangspunkt war die Veröffentlichung von Richter et al., die in ihrer Studie den Effekt einer Telefonintervention bei Typ-2-Diabetiker auf körperliche Aktivität und kardiovaskuläre Risikofaktoren in einem 3-Monatszeitraum untersuchten [7].
Die Autoren dieser Arbeit identifizierten und extrahierten aus der Arbeit von Richter et al. unabhängig voneinander die Textpassagen zu den 25 Punkten der CONSORT 2010 Checkliste. Zusätzlich führten sie eine Bewertung des Berichtens zu dem jeweiligen Punkt durch. Widersprüche wurden durch Gespräche in Konsens umgewandelt [7].

\section{Ergebnisse}

Tab. 1 zeigt die Ergebnisse zur Beurteilung der Zusammenfassung. Die Ergebnisse für die Checkliste des CONSORT 2010 Statements sind in Tab. 2 zusammengestellt.

\section{Diskussion \\ $\nabla$}

Die Interpretation und Bewertung klinischer Studien durch den Leser hängt entscheidend von der Berichtsqualität ab. Im Gegensatz zu dieser scheinbar offensichtlichen Aussage enthielten und enthalten nicht alle Publikationen die Angaben, die zu einer kritischen Bewertung einer Studie notwendig sind [5]. Dieses wurde seit Anfang der 1990er Jahre offensichtlich, als im Rahmen der aufkommenden Evidenzbasierten Medizin systematische Reviews die kritische Bewertung publizierter Studienergebnisse erforderlich machten [1]. Als Konsequenz daraus wurde eine Reihe von Leitlinien für Forschungsberichte entwickelt.

Die Anwendung des CONSORT 2010 Statements auf die Publikation von Richter et al. [7] zeigt, dass sich Leser bei der kritischen Evaluation von Fachartikeln an diesen Leitlinien orientieren können, um die Qualität und Übertragbarkeit einer Studie einzuschätzen. Allerdings muss bei der kritischen Beurteilung eines Artikels der Unterschied zwischen der Qualität des Berichtens und der Qualität der eigentlichen Studie unterschieden werden.

Danksagung: Diese Arbeit entstand während des Forschungsfreisemesters von AZ an dem Institut Montefiore der Universität Lüttich (Belgien). AZ dankt den Kollegen am Institut Montefiore sehr herzlich für die Möglichkeit, dort zu arbeiten. Die Arbeit entstand während der Vorbereitung der Autoren auf den Kursteil Evidenzbasierte Medizin im Rahmen des Kurses Ärztliches Qualitätsmanagement an der Akademie für Fortbildung und Weiterbildung der Landesärztekammer Hessen (Sigrid Blehle, Dr. Roland Kaiser). 
- Die zentralen Leitlinien für Forschungsberichte liegen in deutscher Sprache frei verfügbar vor (https://www.thiemeconnect.de/ejournals/toc/dmw/104011. Sie helfen Autoren von Fachartikeln, vollständige und transparente Berichte zu erstellen.

- Leser können Leitlinien-Elemente zur Einschätzung der Qualität einer publizierten Studie verwenden. Dabei müssen sie zwischen der Qualität des Berichts und der Qualität der Studie unterscheiden.

Autorenerklärung: Die Autoren erhalten ein Honorar von der Akademie für Fortbildung und Weiterbildung der Landesärztekammer Hessen für das Erstellen von Unterlagen für den Kursteil Evidenzbasierte Medizin im Rahmen des Kurses Ärztliches Qualitätsmanagement.

\section{Literaturverzeichnis}

1 Häussler B. Definition, Vorgehensweise und Ziele der evidenzbasierten Medizin. Dtsch Med Wochenschr 2005; 130 Suppl 2: S66-71

2 Hopewell S, Clarke M, Moher D et al. CONSORT for reporting randomised trials in journal and conference abstracts. Lancet 2008; 371: 281-283

3 Hopewell S, Clarke M, Moher D et al. CONSORT for reporting randomized controlled trials in journal and conference abstracts: explanation and elaboration. PLoS Med 2008; 5: e20-

4 Ioannidis JP, Evans SJ, Gøtzsche PC et al. Better reporting of harms in randomized trials: an extension of the CONSORT statement. Ann Intern Med 2004; 141: 781788

5 Meerpohl JJ, Blümle A, Antes G, Elm E. Leitlinien für Forschungsberichte sind auch für Leser medizinischer Fachartikel hilfreich: CONSORT, STARD, STROBE \& Co. Dtsch Med Wochenschr 2009; 134: 2078-2083

6 Moher D, Hopewell S, Schulz KF et al. CONSORT 2010 explanation and elaboration: updated guidelines for reporting parallel group randomised trials. BMJ 2010; 340: c869-

7 Richter H, Kraft K, Kleinwechter $\mathrm{H}$ et al. Telefonintervention bei Typ-2-Diabetikern: Zunahme der körperlichen Aktivität und Reduktion von kardiovaskulären Risikofaktoren. Dtsch Med Wochenschr 2008; 133: 2203-2208

8 Pittler MH, Blümle A, Meerpohl JJ, Antes G. CONSORT 2010: Aktualisierte Leitlinie für Berichte randomisierter Studien im Parallelgruppen-Design. Dtsch Med Wochenschr 2011; 136: e20-e23 
Tab. 1 Zu verwendende Items, wenn über randomisierte Studien in Zeitschriften oder Zusammenfassungen für Tagungen berichtet wird (in Anlehnung an [2]), angewendet auf die Publikation von Richter et al. [7].

\begin{tabular}{|c|c|c|}
\hline Item & Beschreibung & Vorhanden (Bemerkungen) \\
\hline Titel & Identifikation der Studie als randomisierte Studie & $\begin{array}{l}\text { - } \\
\text { (Bis vor wenigen Jahren wurde die Identifikation der Studie als randomisierte Studie nur in } \\
\text { der Zusammenfassung gefordert. Die Studie wird aber in der gesamten Zusammenfassung } \\
\text { nicht als randomisierte Studie identifiziert. Stattdessen entstand bei den Beurteilern beim } \\
\text { Lesen der englischsprachigen Zusammenfassung aufgrund der Formulierung „The patients } \\
\text { in a matching control group ( } n=29 \text { ) were not phoned“ der Eindruck, dass es sich bei der } \\
\text { Studie um eine gematchte Fall-Kontrollstudie handelt.) }\end{array}$ \\
\hline Studiendesign & $\begin{array}{l}\text { Beschreibung des Studiendesigns (z.B. Parallel- } \\
\text { gruppendesign, Cluster-randomisierte Studie, } \\
\text { Nicht-Unterlegenheitsstudie) }\end{array}$ & - \\
\hline \multicolumn{3}{|l|}{ Methoden } \\
\hline - Teilnehmer & $\begin{array}{l}\text { Eignungskriterien für Teilnehmer und die Settings, } \\
\text { in denen die Daten gesammelt wurden (z.B. Uni- } \\
\text { versitätsklinika, Praxen niedergelassener Ärzte) }\end{array}$ & $\begin{array}{l}\text { (Es wurde nur die Information gegeben, dass es Patienten mit Typ-2-Diabetes sind.) } \\
\text { ( }\end{array}$ \\
\hline - Interventionen & Vorgesehene Interventionen für jede Gruppe & $x$ \\
\hline - Fragestellung & Spezifische Fragestellung oder Hypothese & - \\
\hline - Zielkriterium & Klar definierter primärer Endpunkt des Berichts & $\begin{array}{l}\text { - } \\
\text { (Es werden viele Begriffe genannt, wie Bewegungsverhalten, Aktivitätsumfang, verschiede- } \\
\text { ne anthropometrische Variablen sowie Blutfettparameter. Klare Zielkriterien werden nicht } \\
\text { formuliert. Die Parameter wurden auch nicht in den Methoden sondern in den Ergebnissen } \\
\text { genannt) }\end{array}$ \\
\hline - Randomisierung & $\begin{array}{l}\text { Wie Teilnehmer den Behandlungen } \\
\text { zugeteilt wurden }\end{array}$ & $\begin{array}{l}\text { - } \\
\text { (Durch die Formulierung, dass Patienten mittels „Losverfahren“ zugeordnet wurden, sowie } \\
\text { die Tatsache, dass sich zunächst genau gleich viele Personen in den beiden Gruppen befan- } \\
\text { den, wird vermutet, dass es sich um eine trunkierte Randomisierung handelt) }\end{array}$ \\
\hline - Verblindung & $\begin{array}{l}\text { Ob oder ob nicht Teilnehmer, Behandler und } \\
\text { Beurteiler der Zielkriterien im Hinblick auf } \\
\text { Gruppenzuteilung verblindet waren }\end{array}$ & $\begin{array}{l}\text { - } \\
\text { (Dadurch, dass die Behandlung in einem Telefoninterview bestand, konnten die Behandler } \\
\text { nicht verblindet sein) }\end{array}$ \\
\hline \multicolumn{3}{|l|}{ Ergebnisse } \\
\hline $\begin{array}{l}\text {-Anzahl randomi- } \\
\text { siert }\end{array}$ & $\begin{array}{l}\text { Anzahl der Teilnehmer, die in jede Gruppe } \\
\text { randomisiert wurden }\end{array}$ & $x$ \\
\hline - Rekrutierung & Status der Studie & $\begin{array}{l}\text { - } \\
\text { (Es kann aber davon ausgegangen werden, dass die Studie beendet ist) }\end{array}$ \\
\hline - Anzahl analysiert & $\begin{array}{l}\text { Anzahl der Teilnehmer je Gruppe, } \\
\text { die analysiert wurden }\end{array}$ & $\begin{array}{l}\text { - } \\
\text { (In Tab. } 3 \text { werden für jede Analyse die Fallzahlen angegeben) }\end{array}$ \\
\hline -Zielkriterium & $\begin{array}{l}\text { Für den primären Endpunkt, das Ergebnis für } \\
\text { jede Gruppe und die geschätzte Effektstärke } \\
\text { und seine Genauigkeit }\end{array}$ & $\begin{array}{l}\text { - } \\
\text { (Die Ergebnisse wurden nur unspezifisch berichtet: „.... kam es zu einer Abnahme der Para- } \\
\text { meter ..... Es wurden weder p-Werte berichtet, noch Signifikanzen benannt. Effektstärken } \\
\text { und Genauigkeiten wurden nicht berichtet.) }\end{array}$ \\
\hline - Nebenwirkungen & $\begin{array}{l}\text { Wichtige unerwünschte Wirkungen oder } \\
\text { Nebenwirkungen }\end{array}$ & - \\
\hline $\begin{array}{l}\text { Schlussfolgerun- } \\
\text { gen }\end{array}$ & Allgemeine Interpretation der Ergebnisse & $\begin{array}{l}\text { (Da allerdings die Zielkriterien in der Zusammenfassung nicht präzise formuliert wurden } \\
\text { und keine statistischen Zahlen (p-Werte, Effektstärken mit Genauigkeit) berichtet wurden, } \\
\text { lässt sich die Gültigkeit der Schlussfolgerung aus der Zusammenfassung nicht erschließen. }\end{array}$ \\
\hline $\begin{array}{l}\text { Registrierung der } \\
\text { Studie }\end{array}$ & $\begin{array}{l}\text { Registrierungsnummer und Name des } \\
\text { Studienregisters }\end{array}$ & - \\
\hline $\begin{array}{l}\text { Finanzielle Unter- } \\
\text { stützung }\end{array}$ & Quelle der finanziellen Unterstützung & $\begin{array}{l}\text { - } \\
\text { (Zwar geben die Autoren an, dass sie keine finanziellen Verbindungen mit einer Firma ha- } \\
\text { ben, deren Produkt in dem Beitrag eine wichtige Rolle spielt (oder mit einer Firma, die ein } \\
\text { Konkurrenzprodukt vertreibt). Dennoch bleibt offen, wie die Studie finanziert wurde) }\end{array}$ \\
\hline
\end{tabular}


Tab. 2a Punkt für Punkt Betrachtung der Checkliste des CONSORT 2010 Statements [8], angewendet auf die Publikation von Richter et al. [7].

\author{
Item 1a. Titel und Zusammenfassung \\ Beschreibung: Identifikation als randomisierte Studie im Titel. \\ Bemerkung: Weder im Titel noch in der Zusammenfassung wird die Studie als randomisierte Studie identifiziert. \\ Item 1 b. Titel und Zusammenfassung
}

Beschreibung: Strukturierte Zusammenfassung von Studiendesign, Methoden, Resultaten und Schlussfolgerungen (siehe auch CONSORT für Zusammenfassungen [4]) Bemerkung: Die Bewertung der Zusammenfassung ist in Tab. 1 erfolgt.

Item 2a. Einleitung. Hintergrund und Ziele

Beschreibung: Wissenschaftlicher Hintergrund und Begründung der Studie.

S. 2203, Spalten 1 und 2: ... Kognitive und Schulungsansätze waren bisher nicht ausreichend erfolgreich. 90\% der Patienten mit Diabetes mellitus Typ 2 sind adipös oder übergewichtig [30], S. 1: 72\% erreichen nicht das empfohlene Maß an körperlicher Aktivität [23]. Einen Lösungsansatz scheint die, vor allem in den USA seit einigen Jahren praktizierte, Methode der Telefonintervention zu bieten....

Bemerkung: Prägnante, präzise Beschreibung.

Item 2 b. Einleitung. Hintergrund und Ziele

Beschreibung: Genaue Fragestellung oder Hypothesen.

S. 2203, Spalte 2: Im Folgenden wird die Auswirkung einer 3-monatigen Telefonintervention auf Bewegungsverhalten und verschiedene kardiovaskuläre Risikofaktoren bei 41 Patienten mit Diabetes mellitus Typ 2 untersucht.

Bemerkung: Es wird weder eine spezifische Frage noch eine operationalisierte Hypothese formuliert.

Item 3a. Methoden. Studiendesign

Beschreibung: Beschreibung des Studiendesigns (z. B. parallel, faktoriell) einschließlich des Zuteilungsverhältnisses.

S. 2204, Spalte 1: Durch Randomisierung mittels Losverfahren erfolgte die Einteilung in Interventions- und Kontrollgruppe.

Bemerkung: Dieser Formulierung kann man entnehmen, dass es sich um ein Parallelgruppendesign gehandelt hat.

Item 3b. Methoden. Studiendesign

Beschreibung: Wichtige Änderungen der Methoden nach Studienbeginn (wie z. B. Eignungskriterien), mit Gründen.

Bemerkung: Nicht beschrieben. Bis vor Kurzem wurde auch nicht erwartet, dieses zu erwähnen, wenn keine Änderungen in den Methoden nach Studien-

beginn vorgenommen wurden.

Item 4a. Methoden. Studienteilnehmer

Beschreibung: Eignungskriterien der Teilnehmer.

S. 2204, Spalte 1: Einschlusskriterien waren das Vorliegen eines Typ-2-Diabetes, und es durften keine Kontraindikationen gegen sportliche Alltagsbelastungen vorliegen.

Bemerkung: Keine.

Item 4b. Methoden. Studienteilnehmer

Beschreibung: Umgebung und Orte der Studiendurchführung.

S. 2204, Spalte 1: Die Rekrutierung der Probanden erfolgte in 2 Kieler Diabetesschwerpunktpraxen im Rahmen von strukturierten Diabetesschulungsprogrammen ... Die sich direkt an die Schulung anschließende 12-wöchige Intervention bestand lediglich darin, die Probanden ... anzurufen und anhand eines Fragebogens ... zu befragen. Die Befragung wurde immer durch die gleiche Person mit dem gleichen Fragebogen durchgeführt.

Bemerkung: Keine.

\title{
Item 5. Methoden. Interventionen
}

Beschreibung: Durchgeführte Interventionen in jeder Gruppe mit präzisen Details um Studienreplikation zu ermöglichen, einschließlich wie und wann die Interventionen durchgeführt wurden.

P. 2204, Spalte 1: ... im Rahmen von strukturierten Diabetesschulungsprogrammen nach den Empfehlungen der Deutschen Diabetes Gesellschaft (DDG).... Eine Diabetes- und weitere, z. B. antihypertensive Therapien erfolgten unabhängig von der Intervention im Rahmen der üblichen Betreuung durch die Hausarzt oder Diabetesschwerpunktpraxis. Die sich direkt an die Schulung anschließende 12-wöchige Intervention bestand lediglich darin, die Probanden der Interventionsgruppe einmal wöchentlich, die der Kontrollgruppe jeweils einmal in der ersten und in der letzten Woche anzurufen und anhand eines Fragebogens (Info 1) zu ihrem Bewegungsverhalten innerhalb der vergangenen Woche zu befragen. Die Befragung wurde immer durch die gleiche Person mit dem gleichen Fragebogen durchgeführt. Bemerkung: Die verwendete Intervention ist eine komplexe Intervention. Sie besteht aus Diabetesschulung plus Hausarztbetreuung oder Betreuung durch die Diabetesschwerpunktpraxis. Hinzu kommt die Telefonintervention, die die Intervention von Interesse ist.

Zur Diabetesschulung wird erwähnt, dass sie strukturiert nach den Empfehlungen der DDG erfolgte. Es wird nicht von Standardisierungsbemühungen zwischen den beiden Zentren bzw. innerhalb der Zentren berichtet. Standardisierungsbemühungen hinsichtlich der Betreuung durch den Hausarzt bzw. die Diabetesschwerpunktpraxis werden nicht beschrieben. Detaillierte Informationen liegen zur Telefonintervention vor: „Die Befragung wurde immer durch die gleiche Person mit dem gleichen Fragebogen durchgeführt", und der Fragebogen ist im Bericht enthalten.

\section{Item 6a. Methoden. Zielkriterien}

Beschreibung: Vollständig definierte, primäre und sekundäre Endpunkte, einschließlich wie und wann sie erhoben wurden.

S. 2204, Spalte 2, S. 2205, Spalte 1: Nach der standardisierten Schulung (Voruntersuchung) sowie 12 Wochen später (Nachuntersuchung) wurden folgende Daten erhoben: Größe, Gewicht, BMI, Körperfettanteil (Kaliperuntersuchung), Taillenumfang in Höhe des Bauchnabels, arterieller Blutdruck, Nüchternblutzucker, HbA 1 -Wert, HDL, LDL- und Gesamtcholesterin sowie Triglyceride. ... Mehrere voneinander abhängige oder miteinander in Zusammenhang stehende Variablen wurden gleichberechtigt nebeneinander ausgewertet, ...

Bemerkung: Der Begriff Endpunkt wird nicht verwendet. Wann die Endpunkte erhoben wurden, wird beschrieben. Allerdings fehlen detaillierte Beschreibungen, wie die Endpunkte erhoben wurden. 
Tab. 2b Punkt für Punkt Betrachtung der Checkliste des CONSORT 2010 Statements [8], angewendet auf die Publikation von Richter et al. [7].

\section{Item 6b. Methoden. Zielkriterien \\ Beschreibung: Alle Änderungen der Studienendpunkte nach Studienbeginn mit Angabe der Gründe.}

Bemerkung: Keine Beschreibung. Eine klare Beschreibung wäre gewesen: „Es gab keine Änderungen der Zielkriterien nach Studienbeginn. “

Item 7a. Methoden. Fallzahl

Beschreibung: Wie wurde die Fallzahl berechnet.

Bemerkung: Eine Begründung der Fallzahl fehlt, obwohl weder weniger Patienten als erforderlich noch mehr als zwingend notwendig in eine klinische Studie eingeschlossen werden sollten. Auch fehlt eine Angabe der statistischen Macht der Studie.

Item 7b. Methoden. Fallzahl

Beschreibung: Falls zutreffend, Erklärung aller Zwischenanalysen und Stoppregeln.

Bemerkung: Es gibt keine Hinweise zu Zwischenauswertungen oder Stoppregeln.

Item 8a. Methoden. Randomisierung: Erzeugen der Sequenz

Beschreibung: Methode zur Generierung der Zufallszuteilung.

S. 2204, Spalte 1: Durch Randomisierung mittels Losverfahren erfolgte die Einteilung in Interventions- und Kontrollgruppe.

Bemerkung: Das Losverfahren wird nicht näher beschrieben. Aufgrund der ursprünglich identischen Fallzahl in beiden Gruppen könnte vermutet werden, dass eine trunkierte Randomisierung durchgeführt wurde.

\section{Item 8b. Methoden. Randomisierung: Erzeugen der Sequenz}

Beschreibung: Art der Randomisierung; Details aller Restriktionen (z.B. Blockbildung, Blocklänge).

Bemerkung: Hierzu findet sich keine Beschreibung.

\section{Item 9. Methoden. Randomisierung: Geheimhaltung des Zuordnungs-Mechanismus}

Beschreibung: Mechanismen zur Umsetzung der Zuteilungssequenz (z.B. sequenziell nummerierte Behälter) und Beschreibung aller Schritte zur Verdeckung der

Sequenz bis zur Interventionszuordnung.

Bemerkung: Verfahren zur Geheimhaltung der Sequenz werden nicht beschrieben.

Item 10. Methoden. Randomisierung: Implementation

Beschreibung: Wer führte die Zufallszuteilung durch, wer nahm die Teilnehmer in die Studie auf und wer teilte die Teilnehmer den Interventionen zu.

Bemerkung: Diese Aspekte werden in dem Bericht nicht beschrieben.

\section{Item 11a. Methoden. Verblindung}

Beschreibung: Falls durchgeführt, wer bei der Interventionszuordnung verblindet war (z.B. Teilnehmer, Ärzte, Therapeuten, diejenigen, die die Endpunkte beurteilten) und wie. Bemerkung: Zur Verblindung wird nichts berichtet. Die Behandler konnten im Telefoninterview nicht verblindet werden. Eine verblindete Nachuntersuchung hätte sich einfach realisieren lassen und ist möglicherweise erfolgt. In Abhängigkeit des Informationsmaterials und abhängig von einer entsprechenden Zustimmung durch die Ethikkommission hätte die Studie möglicherweise vollständig verblindet realisiert werden können.

\section{Item 11b. Methoden. Verblindung}

Beschreibung: Falls relevant, Beschreibung der Ähnlichkeit der Interventionen.

Bemerkung: Hierzu wird nichts berichtet.

\section{Item 12a. Methoden. Statistische Verfahren}

Beschreibung: Statistische Methoden, die zum Vergleich der Gruppen hinsichtlich primärer und sekundäre Endpunkte eingesetzt wurden.

S. 2205, Spalte 1: Die anderen Parameter wurden mittels Kolmogorov-Smirnov-Anpassungstest auf Normalverteilung geprüft, eine signifikante Abweichung von der Normalverteilung besteht bei $p<0,05$. Bei Normalverteilung wurde mittels Levene-Test auf Homogenität der beiden Stichprobenvarianzen (Telefon- und Kontrollgruppe) getestet. Zur Prüfung auf signifikante Unterschiede zwischen beiden Gruppen wurde der t-Test nach Student für gleiche bzw. ungleiche Varianzen angewandt. Das Signifikanzniveau wurde auf eine Irrtumswahrscheinlichkeit von $p<0,05$ festgelegt. ... Mehrere voneinander abhängige oder miteinander in Zusammenhang stehende Variablen wurden gleichberechtigt nebeneinander ausgewertet, folglich wurde multipel getestet. Das Signifikanzniveau ist nicht entsprechend adjustiert worden. Die Interpretation der Ergebnisse muss also explorativ als Hinweis auf die Effekte der Telefonintervention erfolgen.

Bemerkung: Wie oben beschrieben wurde, unterscheiden die Autoren nicht zwischen primären und sekundären Endpunkten. Es wurde nicht beschrieben, wann der $t$-Test für ungleiche Varianzen verwendet wurde und wann der für gleiche Varianzen. Möglicherweise wurde der Levene-Test als sogenannter Vorschalttest verwendet. Das bedeutet, dass der spezifische t-Test für gleiche bzw. ungleiche Varianzen in Abhängigkeit des p-Werts des Levene-Tests gewählt wurde. Ein solches Vorgehen wäre statistisch inkorrekt, und die beiden verschiedenen t-Tests könnten auch zu unterschiedlichen Ergebnissen führen. Auch bleibt unklar, warum die Autoren auf Normalverteilung getestet haben, wenn ausschließlich der $t$-Test Verwendung findet.

Der letzte Satz „Die Interpretation der Ergebnisse muss also explorativ als Hinweis auf die Effekte der Telefonintervention erfolgen. “ besagt, dass die Studie keine definitiven Schlussfolgerungen zur Wirksamkeit der Telefonintervention erlaubt. In der Zusammenfassung findet sich aber folgende Schlussfolgerung: „Mittels einer wöchentlichen telefonischen Abfrage des Bewegungsverhaltens über 3 Monate kann gesundheitsbewusstes Verhalten gesteigert und das kardiovaskuläre Risikoprofil verbessert werden“. Diese beiden Sätze widersprechen sich.

\section{Item 12b. Methoden. Statistische Verfahren}

Beschreibung: Methoden, die für zusätzliche Analysen eingesetzt wurden, wie Subgruppenanalysen, adjustierte Analysen.

Bemerkung: Hierzu wurde in dem Artikel nichts erwähnt. 
Tab. 2c Punkt für Punkt Betrachtung der Checkliste des CONSORT 2010 Statements [8], angewendet auf die Publikation von Richter et al. [7].

\section{Item 13a. Ergebnisse. Patientfluss (ein Flussdiagramm wird dringend empfohlen)}

Beschreibung: Für jede Gruppe, Anzahl der Studienteilnehmer die randomisiert zugeteilt wurden, die die geplante Intervention erhielten und die hinsichtlich des primären Endpunktes analysiert wurden.

S. 2204, Spalte 1: Nach mündlicher und schriftlicher Information über die Studie erklärten sich 46 der Kursteilnehmer mittels ihrer Unterschrift auf einer Einverständniserklärung zur Teilnahme an der Untersuchung bereit.

... gingen die Daten von 41 Personen in die Auswertung ein.

Bemerkung: Ein Flussdiagramm steht nicht zur Verfügung. Die Information, wie viele Studienteilnehmer randomisiert wurden, wird nicht gegeben. Es sind mutmaßlich die 46 Patienten randomisiert worden, die ihr schriftliches Einverständnis zur Studienteilnahme gaben. Wie viele Patienten auf Eignung zur Studienteilnahme gescreent wurden, wie viele prinzipiell für eine Studienteilnahme geeignet gewesen wären, wie viele nicht an der Studie teilgenommen haben und welche Gründe für die Nichtteilnahme angegeben wurden, wird nicht berichtet.

Die Anzahl der Personen, die in der Endauswertung berücksichtigt wurden, wird berichtet.

\section{Item 13b. Ergebnisse. Patientenfluss (ein Flussdiagramm wird dringend empfohlen)}

Beschreibung: Für jede Gruppe, Zahl der Studienausscheider und Ausschlüsse nach Randomisierung mit Angabe von Gründen.

S. 2204, Spalte 1: Nach mündlicher und schriftlicher Information über die Studie erklärten sich 46 der Kursteilnehmer mittels ihrer Unterschrift auf einer Einverständniserklärung zur Teilnahme an der Untersuchung bereit. ... Nach 2 Studienabbrüchen aus persönlichen organisatorischen Gründen (jeweils eine Person pro Gruppe) und 3 Ausschlüssen aufgrund eines Gestationsdiabetes, eines Bandscheibenvorfalls und des Nichterscheinens zur zweiten Blutentnahme (alle 3 Personen aus der Kontrollgruppe) gingen die Daten von 41 Personen in die Auswertung ein.

Bemerkung: Diese Informationen wurden in den Methoden gegeben. Unklar ist, warum die Person mit Gestationsdiabetes von der Studie ausgeschlossen wurde. Unklar bleibt, ob der Bandscheibenvorfall eine „Kontraindikation gegen sportliche Alltagsbelastungen“ darstellt.

Dass die Person mit fehlender Nachbeobachtung ausgeschlossen wurde, ist nachvollziehbar. Im Sinne einer „intention-to-treat“-Auswertung hätte sie in der Studienpopulation verbleiben sollen.

\section{Item 14a. Ergebnisse. Rekrutierung}

Beschreibung: Zeitraum der Rekrutierung und Nachbeobachtung.

Bemerkung: Daten zum Beginn und Ende der Rekrutierung bzw. der Nachbeobachtung werden nicht gegeben.

Item 14b. Ergebnisse. Rekrutierung

Beschreibung: Warum die Studie endete oder gestoppt wurde.

Bemerkung: Eine Erklärung zum Studienende wird in der Publikation nicht gegeben. Da davon auszugehen ist, dass keine Fallzahlplanung stattfand, wurde die Studie nach Beobachtung der 41 rekrutierten Patienten beendet.

Item 15. Ergebnisse. Ausgangsdaten

Beschreibung: Eine Tabelle demografischer und klinischer Charakteristika für jede Gruppe.

S. 2206: Tabelle 3.

Bemerkung: Keine.

Item 16. Ergebnisse. Anzahl analysiert

Beschreibung: Für jede Gruppe Anzahl der Teilnehmer, die in jede Analyse eingeschlossen wurde und Angabe ob die Analyse den ursprünglich zugeteilten Gruppen entsprach.

S. 2206, Tabelle 3.

Bemerkung: Keine.

Item 17a. Ergebnisse. Zielkriterien und Schätzung

Beschreibung: Für jeden primären und sekundären Endpunkt, Ergebnisse für jede Gruppe und die geschätzte Effektgröße sowie ihre Präzision (z. B. 95\%-Konfidenzintervall).

S. 2206, Tabelle 3.

Bemerkung: Trotz der Ausführlichkeit der Tabelle werden weder p-Werte noch Konfidenzintervalle berichtet. Allerdings beinhaltet die Tabelle gruppenspezifische Mittelwerte und Standardabweichungen zusammen mit der Information, ob der $p$-Wert $<0,05$ ist.

Item 17b. Ergebnisse. Zielkriterien und Schätzung

Beschreibung: Für binäre Zielkriterien ist die Angabe von absoluten und relativen Effektgrößen empfohlen.

S. 2206, Tabelle 3.

Bemerkung: Es werden gruppenspezifische absolute und relative Häufigkeiten berichtet, aus denen sich alle relevanten Effektgrößen herleiten lassen können.

Item 18. Ergebnisse. Zusätzliche Analysen

Beschreibung: Resultate von allen weiteren Analysen, einschließlich Subgruppenanalysen und adjustierten Analysen mit Angabe, ob diese vordefiniert oder exploratorisch durchgeführt wurden.

Bemerkung: Hierzu wird in der Publikation nichts berichtet.

Item 19. Ergebnisse. Nebenwirkungen

Beschreibung: Alle wichtigen Schäden und unerwünschten Wirkungen in jeder Gruppe (siehe CONSORT für Schaden [4]).

Bemerkung: Hierzu wird in der Publikation nichts berichtet. 
Tab. 2d Punkt für Punkt Betrachtung der Checkliste des CONSORT 2010 Statements [8], angewendet auf die Publikation von Richter et al. [7].

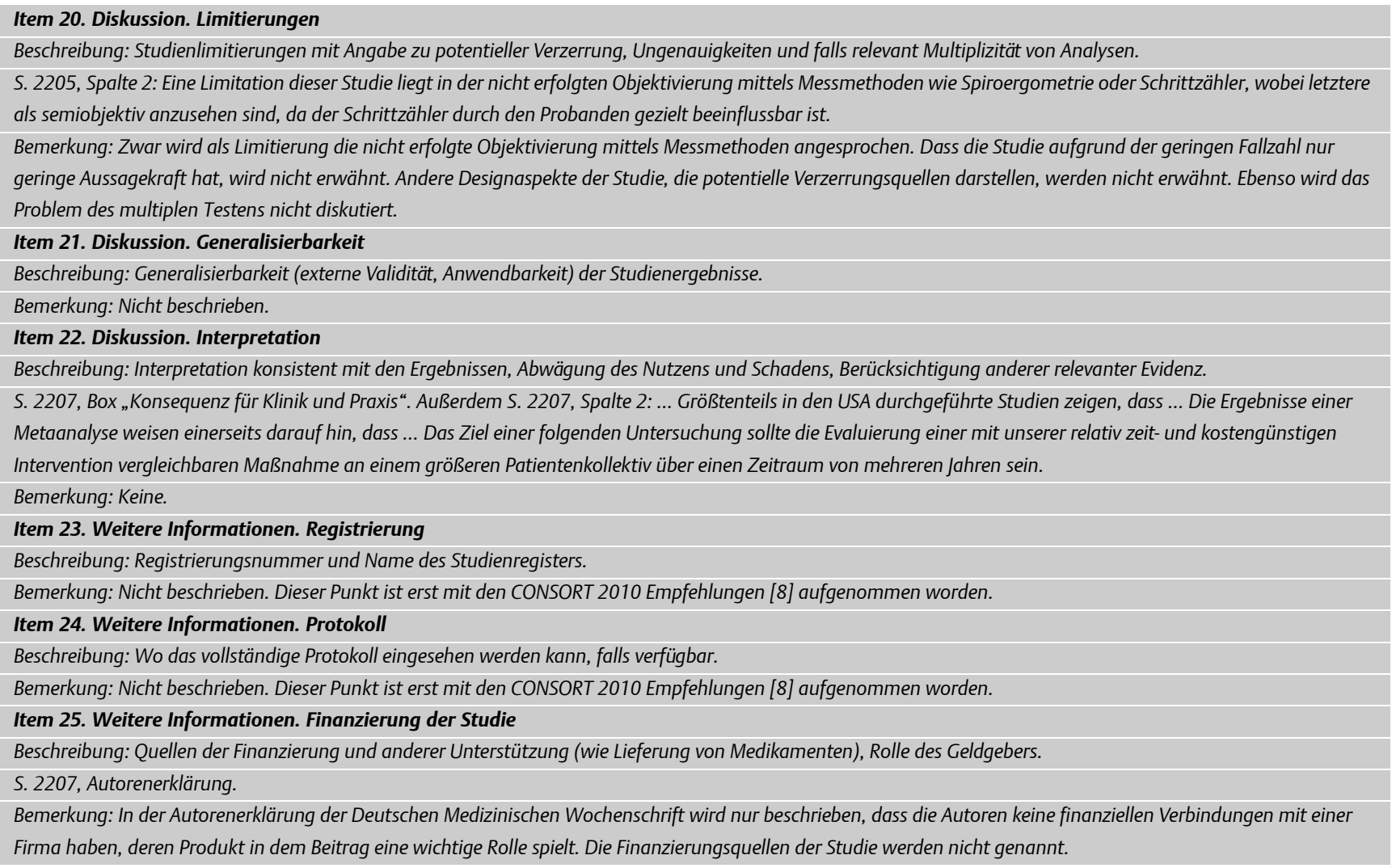

